\title{
Outcomes of primary sleeve gastrectomy versus conversion sleeve gastrectomy in morbidly obese patients
}

\author{
Jong Seob Park', Sang-Moon Han² \\ ${ }^{1}$ Department of Surgery, Myongji Hospital, Goyang, Korea \\ ${ }^{2}$ Department of Surgery, CHA Gangnam Medical Center, CHA University School of Medicine, Seoul, Korea
}

\begin{abstract}
Purpose: Our aim for this study was to evaluate early and late complications and outcomes of primary sleeve gastrectomy (PSG) versus conversion sleeve gastrectomy (CSG).

Methods: From February 2013 to December 2016, a total of 180 patients underwent sleeve gastrectomy 1150 PSG and 30 CSGI. All patients received a metal clipping at the end of the stapling line and a continuous seromuscular suture at the resection margin, for reinforcement.

Results: There were no differences in the percentages among males and females or age between the 2 groups, but the body mass index (BMI) of the PSG group was higher at $36.8 \pm 4.7$ than that of the CSG group $(32.4 \pm 5.7, P<0.001)$. Three early postoperative complications were noted in the PSG group; 1 patient underwent repeat laparoscopic exploration due to pancreatic injury, and 2 other patients developed pulmonary atelectasis. On the contrary, 2 early minor complications were noted in the CSG group. Thirty-eight patients (25.3\%) in the PSG group developed 43 late, minor complications, while 9 patients (30.0\%) developed 11 late minor and 1 major complication in the CSG group. However, there was no difference in complication rate between PSG and CSG. Percentage excess BMI loss at 3, 6, and 12 months after surgery was comparable between the groups.

Conclusion: PSG and CSG were comparable in terms of postoperative complications and loss of weight. Therefore, CSG could be used for failed primary restrictive bariatric surgery. However, the durability of these outcomes remains unknown.

[Ann Surg Treat Res 2019;96(5):259-265]
\end{abstract}

Key Words: Bariatric surgery, Reoperation

\section{INTRODUCTION}

Adjustable gastric banding (AGB) is simple to perform, reversible, and safe [1]. However, AGB can cause numerous complications related to the band itself, including slippage, erosion, migration, and esophageal dilatation, due to gastric outlet obstruction or stenosis [2,3]. According to a nationwide study, the removal rate of AGB at 5,6 , and 7 years was $28 \%, 34 \%$, and $40 \%$, respectively, and the conversion rate at 7 years was
$71 \%$ [4]. Sleeve gastrectomy (SG) is superior to AGB in terms of weight loss and impact on obesity related comorbidities [5,6]. As a result, there has been a significant decrease in the number of AGBs performed worldwide, in favor of Roux-en-Y gastric bypass and SG [5-7]. However, even in patients with SG, inadequate weight loss, regaining weight, or complications such as severe gastroesophageal reflux disease, often require revisional bariatric strategies [8]. Revisional or conversion procedures for failed primary bariatric surgery have increased from nearly $0 \%$
Received July 23, 2018, Revised December 11, 2018,

Accepted January 14, 2019

\section{Corresponding Author: Sang-Moon Han}

Department of Surgery, CHA Gangnam Medical Center, CHA University School of Medicine, 566 Nonhyeon-ro, Gangnam-gu, Seoul 06135, Korea

Tel: +82-2-3468-3369, Fax: +82-2-3468-3507

E-mail: surgeryhan@gmail.com

ORCID code: https://orcid.org/0000-0001-8631-0998
Copyright (C) 2019, the Korean Surgical Society

(c) Annals of Surgical Treatment and Research is an Open Access Journal. All articles are distributed under the terms of the Creative Commons Attribution NonCommercial License (http://creativecommons.org/licenses/by-nc/4.0/) which permits unrestricted non-commercial use, distribution, and reproduction in any medium, provided the original work is properly cited. 
in 2008 to about 5\% in 2014 as per a premier database in the United States [7].

SG is one of the options for revisional bariatric surgery. The risk of postoperative complications in patients undergoing revision surgery is higher than that in patients undergoing primary sleeve gastrectomy (PSG) $[9,10]$. On the other hand, several reports have concluded that SG as a revision procedure is feasible and safe. Moreover, additional weight loss and further resolution of comorbidity appear achievable [5,11]. However, there is still debate about whether SG as a revisional or conversion surgery is safe and effective.

This study aimed to compare the early and late complications, and outcomes of PSG with those of conversion laparoscopic sleeve gastrectomy (CSG).

\section{METHODS}

From February 2013 to December 2016, 180 patients who underwent PSG or CSG were enrolled in this study; 150 patients underwent PSG and 30 patients underwent CSG at a minimally invasive obesity surgery center. The indications for CSG included failure to lose weight, gastric band complications (stenosis, erosion, slippage, and infection), and the patient's voluntary choice after previous primary bariatric surgery.

This study was a retrospective analysis of our prospectively collected database. The following demographic data were collected and analyzed: age, sex, body mass index (BMI), comorbidities, the type of primary bariatric surgery, reason for conversion, interval between primary and repeat surgery in CSG, duration of follow-up, duration of surgery, estimated blood loss, simultaneous operation, mean duration of hospital stay, short-term and long-term postoperative complications, and change in the percentage of excess BMI loss (\%EBMIL). Percentage of excess BMI loss is derived by dividing the change in BMI from excess BMI at baseline, which was obtained by subtracting the ideal BMI $\left(23 \mathrm{~kg} / \mathrm{m}^{2}\right)$ from the actual BMI. Since the normal range of BMI in adult Asians is $18.5-22.9 \mathrm{~kg} /$ $\mathrm{m}^{2}$, the ideal BMI cutoff is $23 \mathrm{~kg} / \mathrm{m}^{2}$ [12]. Weight loss failure was defined as percentage of excess weight loss (\%EWL) $<50 \%$ during the first 1 year after bariatric surgery, and weight regain was diagnosed if $\%$ EWL reached $<50 \%$ postoperatively from nadir. Surgical complications were defined as early and late complications, with the cutoff being 30 days after surgery. This study was approved by the Gangnam CHA Medical Center Institutional Review Board (approval number: GCI-16-20).

The surgical procedures for PSG and CSG are not very different. Two 12-mm ports, including one port for the camera, and two 5-mm ports were used, and the Endo-GIA staplers (Ethicon Endo-Surgery, Cincinnati, OH, USA) were applied for SG, following which a continuous seromuscular reinforcement suture at the resection margin was applied.
A 36F bougie was used for resection. A point on the greater curvature of the stomach approximately $4 \mathrm{~cm}$ proximal to the pylorus was used as the distal resection point $[12,13]$. One-stage surgery, performed with band-removal and sleeve gastrectomy simultaneously, was planned for patients who did not suffer from complications of the gastric band, such as erosion, stenosis, slippage, or infection. For the patients who underwent CSG due to complications of the gastric band, a 2-stage surgery was planned. The first step was band removal, followed by SG in the second step. The purpose of our study was explained to the patients, and informed consent was obtained.

All patients underwent a routine gastrografin upper gastrointestinal study on postoperative day 1 . If the outcome was normal, patients received a clear liquid diet and progressed to a full liquid diet for 1 week. Subsequently, a soft diet was followed for 2 weeks and then advanced to a regular diet in the fourth week. Follow-up visits were scheduled every 3 months in the first postoperative year. Later, follow-up visits were scheduled every 6 months. Telephonic interviews were conducted to obtain information on sought postoperative weight loss and general health status, in patients who were lost to follow-up [12].

Data were analyzed by descriptive statistical methods with the SPSS ver. 18.0 (SPSS Inc., Chicago, IL, USA). They were then presented either as means \pm standard deviations or as percentages. The significance of differences between the groups was evaluated using a chi-square test, Fisher exact test, Student t-test or Mann-Whitney test, as appropriate. A P-value $\leq 0.05$ was considered statistically significant.

\section{RESULTS}

There were no differences in sex and age at baseline, between the 2 groups. However, the BMI of the PSG group was higher at $36.8 \pm 4.7$ versus $32.4 \pm 5.7$ of the CSG group $(P<0.001)$. Significant comorbidities in the PSG group compared to the CSG group included fatty liver (50.0\% vs. 26.7\%, P = 0.019), dyslipidemia (50.7\% vs. $16.7 \%, \mathrm{P}=0.001)$, and hypertension (48.0\% vs. $16.7 \%, \mathrm{P}=0.002$ ). The incidence of gallbladder stone was higher in the CSG group than in the PSG group $(4.0 \%$ vs. $20.0 \%, \mathrm{P}=0.006$ ).

Twenty-six patients (86.7\%) underwent primary AGB, 3 patients (10.0\%) underwent PSG, and 1 patient (3.3\%) underwent greater curvature gastric placation after AGB, prior to CSG (Table 1). Among the 26 patients (86.7\%) who underwent CSG after AGB, 9 and 17 patients underwent 1-step CSG and 2-step CSG with gastric band removal, respectively. The indications for conversion were failure to lose weight in 19 patients (63.3\%), and complications of gastric banding in 9 patients (30.0\%), which included 4 band erosions (13.3\%), 1 stenosis (3.3\%), 3 slippages (10.0\%), and 1 infection (3.3\%). The interval of repeat surgery 
Table 1. Patients' general characteristics

\begin{tabular}{|c|c|c|c|}
\hline Characteristic & PSG $(n=150)$ & $\operatorname{CSG}(n=30)$ & P-value \\
\hline Age (yr) & $33.3 \pm 8.0$ & $35.6 \pm 6.5$ & 0.139 \\
\hline \multicolumn{4}{|l|}{ Sex } \\
\hline Female & $127(84.7)$ & $28(93.3)$ & 0.210 \\
\hline Male & $23(15.3)$ & $2(6.7)$ & \\
\hline Weight (kg) & $99.6 \pm 17.1$ & $87.1 \pm 21.2$ & 0.001 \\
\hline Height $(\mathrm{cm})$ & $164.3 \pm 0.1$ & $163.2 \pm 0.1$ & 0.497 \\
\hline Body mass index $\left(\mathrm{kg} / \mathrm{m}^{2}\right)$ & $36.8 \pm 4.7$ & $32.4 \pm 5.7$ & $<0.001$ \\
\hline \multicolumn{4}{|l|}{ Comorbidities $^{\text {a) }}$} \\
\hline Fatty liver & $75(50.0)$ & $8(26.7)$ & 0.019 \\
\hline Dyslipidemia & $76(50.7)$ & $5(16.7)$ & 0.001 \\
\hline Hypertension & $72(48.0)$ & $5(16.7)$ & 0.002 \\
\hline Hiatal hernia & $61(40.7)$ & $9(30.0)$ & 0.274 \\
\hline Insulin resistance & $41(27.3)$ & $5(16.7)$ & 0.221 \\
\hline Obstructive sleep apnea & $33(22.0)$ & $2(6.7)$ & 0.053 \\
\hline Type II diabetes mellitus & $33(22.0)$ & $3(10.0)$ & 0.134 \\
\hline Arthritis \& back pain & $26(17.3)$ & $1(3.3)$ & 0.052 \\
\hline Dys- or amenorrhea & $27(18.0)$ & $2(6.7)$ & 0.174 \\
\hline Reflux esophagitis & $23(15.3)$ & $4(13.3)$ & $>0.999$ \\
\hline Gout & $13(8.7)$ & $2(6.7)$ & $>0.999$ \\
\hline Gallbladder stone & $6(4.0)$ & $6(20.0)$ & 0.006 \\
\hline Depressive disorder & $7(4.7)$ & $1(3.3)$ & $>0.999$ \\
\hline Asthma & $8(5.3)$ & $0(0)$ & 0.356 \\
\hline \multicolumn{4}{|l|}{ Previous bariatric surgery } \\
\hline Gastric banding & - & $26(86.7)$ & \\
\hline One-step conversion surgery & & $9(34.6)$ & \\
\hline Two-step conversion surgery & & $17(65.4)$ & \\
\hline Sleeve gastrectomy & - & $3(10.0)$ & \\
\hline Banding $\rightarrow$ gastric plication & - & $1(3.3)$ & \\
\hline \multicolumn{4}{|l|}{ Reason of conversion operation } \\
\hline Failure of weight loss & - & $19(63.3)$ & \\
\hline Complications of gastric band & & $9(29.9)$ & \\
\hline Erosion & - & $4(13.3)$ & \\
\hline Stenosis & - & $1(3.3)$ & \\
\hline Slippage & - & $3(10.0)$ & \\
\hline Infection & - & $1(3.3)$ & \\
\hline Wanted & - & $2(6.7)$ & \\
\hline Interval of reoperation (mo) & - & $47.3 \pm 31.4$ & \\
\hline Period of follow-up (mo) & $14.2 \pm 10.8$ & $61.0 \pm 31.7$ & $<0.001$ \\
\hline
\end{tabular}

Values are presented as mean \pm standard deviation or number $(\%)$.

PSG, primary sleeve gastrectomy; CSG, conversion sleeve gastrectomy.

a) Included in duplication.

in CSG was $47.3 \pm 31.4$ months. The mean period of follow-up was longer in the CSG group than in the PSG group (14.2 \pm 10.8 months vs. $61.0 \pm 31.7$ months, $\mathrm{P}<0.001$ ) (Table 1).

All 180 patients underwent laparoscopic surgery. The mean duration of surgery was $136.0 \pm 31.8$ minutes in the PSG group versus $217.6 \pm 44.1$ minutes in the CSG group $(\mathrm{P}<0.001)$ (Table 2). Estimated blood loss was lower in the PSG group than in the CSG group $(60.5 \pm 135.3 \mathrm{~mL}$ vs. $135.9 \pm 210.8 \mathrm{~mL}, \mathrm{P}=0.083)$. In 1 patient with previous band erosion, a foreign body, which was a part of the gastric band material, was found incidentally during CSG. Mean hospital stay was lower in the PSG group than in the CSG group (3.6 \pm 1.0 vs. $4.8 \pm 1.8$, respectively, $\mathrm{P}=$ 0.002) (Table 2).

Three early postoperative complications were noted in the PSG group, 2 patients developed pulmonary atelectasis, and Clavien-Dindo surgical complication classification (C-D grade) was grade I [14]. Another patient underwent repeat laparoscopic exploration on postoperative day 2, due to pancreatic injury from the laparoscopic energy device. Two complications occurred in the CSG group. One patient developed pleural 
effusion (C-D grade I), and another patient developed a gastric stricture (C-D grade II) [14]. The patient with the gastric stricture was readmitted thrice after CSG. Each admission lasted 3-4 days, during which she received conservative management. A gastrografin upper gastrointestinal series did not demonstrate any disturbance in the flow of the dye; however, a small phytobezoar was found in the remnant stomach during gastroscopy. The patient remained symptomfree at her 3-year follow-up visit. No patients developed leakage after CSG, and the mortality rate was zero. Thirty-eight patients (25.3\%) in the PSG group developed 43 minor late complications, while 9 patients (30.0\%) in the CSG group developed 11 minor and 1 major late complication. There were no differences in late complications between the groups $(P=0.219)$. One patient in the CSG group underwent adhesiolysis surgery 40 days after the CSG procedure (Table 3).

The results of \%EBMIL at postoperative 3 months $(79.9 \pm$ 34.8) versus 6 months $(107.8 \pm 47.0)$, and 6 months versus 12 months $(124.9 \pm 56.0)$ in the PSG group significantly increased $(\mathrm{P}<0.001$ and $\mathrm{P}<0.001$, respectively). The results in the CSG group were also significantly different $(87.1 \pm 46.8$ at 3 months, $109.8 \pm 54.6$ at 6 months, and $136.6 \pm 55.5$ at 12 months; 3 months vs. 6 months $P<0.001$, and 6 months vs. 12 months $\mathrm{P}<0.001$, respectively). The \%EBMIL at postoperative 3, 6 , and 12 months after surgery was not significantly different between the 2 groups (Table 4).

Table 2. Intraoperative and postoperative features

\begin{tabular}{lccc}
\hline \multicolumn{1}{c}{ Characteristic } & PSG $(\mathrm{n}=150)$ & CSG $(\mathrm{n}=30)$ & P-value \\
\hline Operative time (min) & $136.0 \pm 31.8$ & $217.6 \pm 44.1$ & $<0.001$ \\
Estimated blood loss $(\mathrm{mL})$ & $60.5 \pm 135.3$ & $135.9 \pm 210.8$ & 0.083 \\
Simultaneous operation & & $6(20.0)$ & 0.001 \\
None & $78(52.0)$ & $9(30.0)$ & 0.336 \\
Hiatal hernia repair & $59(39.3)$ & $0(0)$ & $>0.999$ \\
Hiatal hernia \& cholecystectomy & $1(0.7)$ & $0(0)$ & $>0.999$ \\
Hiatal hernia \& liver biopsy & $1(0.7)$ & $5(16.8)$ & 0.603 \\
Liver biopsy & $7(4.7)$ & $4(13.3)$ & 0.007 \\
Cholecystectomy & $4(2.6)$ & $4(13.3)$ & $1(3.3)$ \\
Band removal only & - & $1(3.3)$ & 0.002 \\
Band removal \& hiatal hernia repair & - & - & $4.8 \pm 1.8$ \\
Band removal \& cholecystectomy & - & $3.6 \pm 1.0$ & \\
Foreign body removal ${ }^{\text {a) }}$ & & & \\
Mean hospital stay (day) &
\end{tabular}

Values are presented as mean \pm standard deviation or number (\%).

PSG, primary sleeve gastrectomy; CSG, conversion sleeve gastrectomy.

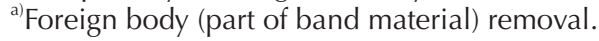

Table 3. Postoperative complications

\begin{tabular}{lccr}
\hline \multicolumn{1}{c}{ Complication } & PSG $(\mathrm{n}=150)$ & CSG $(\mathrm{n}=30)$ & P-value \\
\hline Early postoperative complications ${ }^{\mathrm{a})}(\leq 30$ days $)$ & $3(2.0)$ & $2(6.6)$ & 0.194 \\
Atelectasis (I) & $2(1.3)$ & $0(0)$ & $>0.999$ \\
Pancreatic injury (IIIb) & $1(0.7)$ & $0(0)$ & $>0.999$ \\
Pleural effusion (I) & $0(0)$ & $1(3.3)$ & 0.167 \\
Gastric stricture (II) & $43(28.7)$ & $12(40.0)$ & 0.167 \\
Late postoperative complications (>30 days) & $30(20.0)$ & $6(20.0)$ & $>0.219$ \\
GERD (II) & $4(2.7)$ & $2(6.7)$ & 0.999 \\
Anemia (II) & $5(3.3)$ & $1(3.3)$ & 0.330 \\
Gallbladder stone (II) & $4(2.7)$ & $1(3.3)$ & $>0.999$ \\
Ureter stone (II) & $0(0)$ & & 0.167 \\
Trocar site hernia (IIIb) & &
\end{tabular}

Values are presented as number (\%).

PSG, primary sleeve gastrectomy; CSG, conversion sleeve gastrectomy; GERD, gastroesophageal reflux disease.

${ }^{a}$ According to Clavien-Dindo surgical complication classification. 
Table 4. Changes in percentage of excess body mass index loss

\begin{tabular}{lrrc}
\hline & PSG $(\mathrm{n}=150)$ & CSG $(\mathrm{n}=30)$ & P-value \\
\hline 3 Months & $79.9 \pm 34.8$ & $87.1 \pm 46.8$ & 0.490 \\
6 Months & $107.8 \pm 47.0$ & $109.8 \pm 54.6$ & 0.862 \\
12 Months & $124.9 \pm 56.0$ & $136.6 \pm 55.5$ & 0.401 \\
\hline
\end{tabular}

Values are presented as mean \pm standard deviation or number (\%).

PSG, primary sleeve gastrectomy; CSG, conversion sleeve gastrectomy.

\section{DISCUSSION}

Our results indicate that there were no differences in postoperative early and late complications, and weight loss after PSG and CSG. Duration of surgery, estimated blood loss, and hospital stay were higher or longer in the CSG group than in the PSG group, owing to other simultaneous procedures in some cases. In PSG, 39.3\% of the surgeries included hiatal hernia repair simultaneously with the primary surgery, while nine patients in the conversion surgery underwent a 1-step surgery, which included removal of AGB and CSG simultaneously. Therefore, a head-to-head comparison of the duration of surgery between the 2 groups does not lead to a definite conclusion. Although the duration of surgery, estimated blood loss, and hospital stay were higher or longer in the conversion patients, these are acceptable since these were not associated with significant complications.

The risk of postoperative complications is higher in patients who undergo revision surgery, and is even higher after multiple revisions [9]. The leak rate was reportedly higher after revision of AGB into SG - this procedure involves stapling over the scarred tissue, a longer stapler line, and dissection at the left crus, which can jeopardize the blood supply at the gastroesophageal junction [9,15-17]. However, the surgeon's experience with a number of revisional bariatric procedures decreases risk and complications to an acceptable rate [18-20]. In our institution, appropriate staplers were chosen according to the thickness of each individual patient's stomach, and the time of holding tissue with a stapler before firing should be sufficient for preventing leakage. Accurate angle of the stapler and sufficient mobilization of the stomach from adjacent structures are necessary to prevent creating dog ears at the overlap between the staplings. If staple debris is found at the end of the stapling line before the next stapler firing, it should be removed to prevent another misfired staple. We applied a 5-mm clip to prevent bleeding or leakage at the beginning and end of the stapling line (Fig. 1). Subsequently, a continuous seromuscular suture at the resection margin was applied (Fig. 2). These meticulous steps prevent leakage or bleeding. No leakage

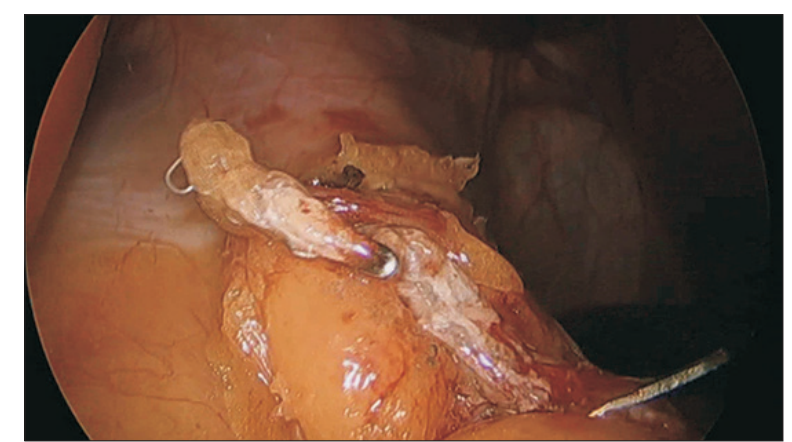

Fig. 1. Metal clipping at each end of stapling line.

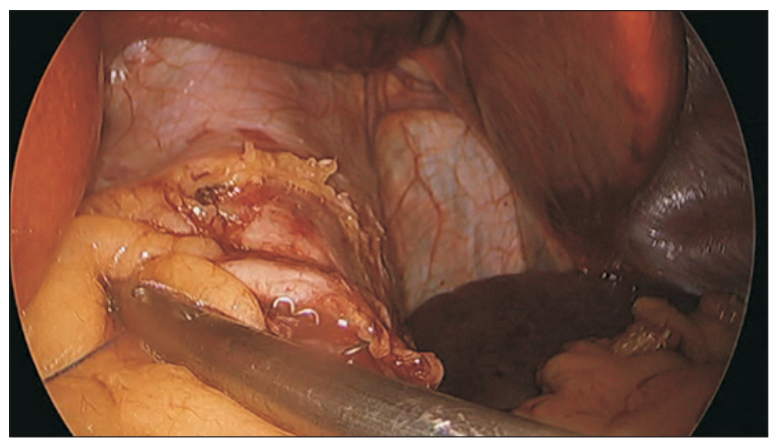

Fig. 2. Continuous seromuscular reinforcement suture at resection margin.

or bleeding was noted in either PSG or CSG group in our study.

Several studies reported that 2-step conversion of failed AGB to $S G$, results in a significantly reduced rate of postoperative staple line leaks, gastric tube stricture, and respiratory complications, and 1-step conversion of gastric band to SG is associated with increased postoperative complications [21,22]. On the contrary, one systematic review and meta-analysis suggests that immediate or delayed revisional bariatric surgeries are both safe options for AGB revisions. There were statistically similar rates of complications, abscess formation $(P=0.54)$, postoperative bleeding $(P=0.77)$, leak and fistula $(P=0.26)$, and morbidity $(P=0.56)$, between 1-step and 2-step surgeries. No mortality events were recorded [23]. Nine patients who underwent 1-step conversion in our study did not develop any early surgical complication. Two early complications, such as pleural effusion and gastric stricture, were noted in the patients who underwent 2-step CSG from AGB. A drawback of the 2-step surgery is that weight could be regained in the interval between band removal and conversion surgery [24]. Therefore, if the tissue at the resection line is healthy, a 1-step conversion surgery should be considered.

Revisional or conversion surgery is expected to increase significantly, considering the annual rate of bariatric surgery in Korea; the rate of revisional surgery in bariatric surgery has increased since $2008[7,25]$. The reasons for conversion sur- 
gery from primary AGB include complications of the gastric band, and regaining of weight. In the early days of bariatric surgery, AGB was performed in over $50 \%$ of the cases $[7,25]$. According to the national data, the band survival rate after AGB was $72 \%$ at 5 years, and about half of the patients underwent revisional surgery. Among several revisional surgeries, SG was the most common revisional procedure (44.3\%) [4]. Thus, the complications of gastric band and weight regain after AGB are likely to increase with time, leading to an increase in conversion surgery.

The results of \%EBMIL at 3 months, 6 months, and 12 months postoperatively were comparable, and good in both groups. These results are in line with those of previous studies [18,2628]. Some studies showed that the rate of weight loss during the early period until 1 year after PSG was better than that after CSG; however, weight loss was similar in both groups at 1 or 2 years after surgery $[26,27]$. The most plausible explanation is the fact that the LAGB to LSG group includes patients that evolve more rapidly toward failure as they have already undergone the experience of surgery-induced restriction and have developed eating habits to overcome the effects of surgery [26]. Our result was different from those of these studies. The patterns of weight loss at postoperative months 3, 6, and 12 in our study were similar between the groups, confirming that PSG and CSG have similar outcomes [28].

Limitations of this study include clinicopathologic differences between the 2 groups, the patients included from a singleinstitution with a small sample size, and retrospective nature of the study. The patients in the PSG group had a higher BMI $(\mathrm{P}<0.001)$ and higher rate of comorbidities like fatty liver $(\mathrm{P}=$ $0.019)$, dyslipidemia $(P=0.001)$, and hypertension $(P=0.002)$. However, as it is related with the results of primary bariatric surgery, it can be a difference in terms in relation thereto.

PSG and CSG are comparable in terms of complications and weight loss. Therefore, CSG could be a strategy for conversion operations after failed primary restrictive bariatric surgery. However, the durability of these outcomes remains unknown.

\section{CONFLICTS OF INTEREST}

No potential conflict of interest relevant to this article was reported.

\section{REFERENCES}

1. Favretti F, Segato G, Ashton D, Busetto L, De Luca M, Mazza M, et al. Laparoscopic adjustable gastric banding in 1,791 consecutive obese patients: 12-year results. Obes Surg 2007:17:168-75.

2. DeMaria EJ, Sugerman HJ, Meador JG, Doty JM, Kellum JM, Wolfe L, et al. High failure rate after laparoscopic adjustable silicone gastric banding for treatment of morbid obesity. Ann Surg 2001;233:80918.

3. Suter M, Calmes JM, Paroz A, Giusti V. A 10-year experience with laparoscopic gastric banding for morbid obesity: high long-term complication and failure rates. Obes Surg 2006;16:829-35.

4. Lazzati A, De Antonio M, Paolino L, Martini F, Azoulay D, Iannelli A, et al. Natural history of adjustable gastric banding: lifespan and revisional rate: a nationwide study on administrative data on 53,000 patients. Ann Surg 2017;265:439-45.

5. Sharples AJ, Charalampakis V, Daskalakis M, Tahrani AA, Singhal R. Systematic review and meta-analysis of outcomes after revisional bariatric surgery following a failed adjustable gastric band. Obes Surg 2017:27:2522-36.

6. Hutter MM, Schirmer BD, Jones DB, Ko CY, Cohen ME, Merkow RP, et al. First report from the American College of Surgeons Bariatric Surgery Center Network: laparoscopic sleeve gastrectomy has morbidity and effectiveness positioned between the band and the bypass. Ann Surg 2011:254:410-20.

7. Abraham A, Ikramuddin S, Jahansouz C, Arafat F, Hevelone N, Leslie D. Trends in bariatric surgery: procedure selection, revisional surgeries, and readmissions. Obes Surg 2016;26:1371-7.

8. Kowalewski PK, Olszewski R, Waledziak MS, Janik MR, Kwiatkowski A, GalazkaSwiderek N, et al. Long-term outcomes of laparoscopic sleeve gastrectomy-a singlecenter, retrospective study. Obes Surg 2018;28:130-4.

9. Hallowell PT, Stellato TA, Yao DA, Robin- son A, Schuster MM, Graf KN. Should bariatric revisional surgery be avoided secondary to increased morbidity and mortality? Am J Surg 2009;197:391-6.

10. Fulton C, Sheppard C, Birch D, Karmali S, de Gara C. A comparison of revisional and primary bariatric surgery. Can J Surg 2017; 60:205-11.

11. Berende CA, de Zoete JP, Smulders JF, Nienhuijs SW. Laparoscopic sleeve gastrectomy feasible for bariatric revision surgery. Obes Surg 2012;22:330-4.

12. Hong JS, Kim WW, Han SM. Five-year results of laparoscopic sleeve gastrectomy in Korean patients with lower body mass index $\left(30-35 \mathrm{~kg} / \mathrm{m}^{2}\right)$. Obes Surg 2015;25: 824-9.

13. Park JS, Han SM. Feasibility and safety of conversion sleeve gastrectomy after failed primary adjustable gastric banding or sleeve gastrectomy. J Metab Bariatr Surg 2016;5:62-6.

14. Dindo D, Demartines N, Clavien PA. Classification of surgical complications: a 
new proposal with evaluation in a cohort of 6336 patients and results of a survey. Ann Surg 2004:240:205-13.

15. Spivak H, Beltran OR, Slavchev P, Wilson EB. Laparoscopic revision from LAP-BAND to gastric bypass. Surg Endosc 2007;21: 1388-92.

16. Iannelli A, Schneck AS, Ragot E, Liagre A, Anduze Y, Msika S, et al. Laparoscopic sleeve gastrectomy as revisional procedure for failed gastric banding and vertical banded gastroplasty. Obes Surg 2009; 19:1216-20.

17. van Wageningen B, Berends FJ, Van Ramshorst B, Janssen IF. Revision of failed laparoscopic adjustable gastric banding to Roux-en-Y gastric bypass. Obes Surg 2006; 16:137-41.

18. Mahawar KK, Graham Y, Carr WR, Jennings N, Schroeder N, Balupuri S, et al. Revisional Roux-en-Y gastric bypass and sleeve gastrectomy: a systematic review of comparative outcomes with respective primary procedures. Obes Surg 2015;25: 1271-80.

19. Abdelgawad M, De Angelis F, Iossa A, Rizzello M, Cavallaro G, Silecchia G. Management of complications and outcomes after revisional bariatric surgery: 3-year experience at a bariatric center of excellence. Obes Surg 2016;26:2144-9.

20. Kirshtein B, Kirshtein A, Perry Z, Ovnat A, Lantsberg L, Avinoach E, et al. Laparoscopic adjustable gastric band removal and outcome of subsequent revisional bariatric procedures: a retrospective review of 214 consecutive patients. Int J Surg 2016;27:133-7.

21. Schneck AS, Lazzati A, Audureau E, Hemery F, Gugenheim J, Azoulay D, et al. One or two steps for laparoscopic conversion of failed adjustable gastric banding to sleeve gastrectomy: a nationwide French study on 3357 morbidly obese patients. Surg Obes Relat Dis 2016;12:840-8.

22. Dietch ZC, Schirmer BD, Hallowell PT. Simultaneous conversion of gastric band to sleeve gastrectomy is associated with increased postoperative complications: an analysis of the American College of Surgeons National Surgical Quality Improvement Program. Surg Endosc 2017;31:522833.

23. Dang JT, Switzer NJ, Wu J, Gill RS, Shi X, Thereaux J, et al. Gastric band removal in revisional bariatric surgery, one-step versus two-step: a systematic review and meta-analysis. Obes Surg 2016;26:866-73.
24. Lanthaler M, Strasser S, Aigner F, Margreiter $\mathrm{R}$, Nehoda $\mathrm{H}$. Weight loss and quality of life after gastric band removal or deflation. Obes Surg 2009;19:1401-8.

25. Lee HJ, Ahn HS, Choi YB, Han SM, Han SU, Heo YS, et al. Nationwide survey on bariatric and metabolic surgery in Korea: 2003-2013 results. Obes Surg 2016;26:6915.

26. Noel P, Schneck AS, Nedelcu M, Lee JW, Gugenheim J, Gagner M, et al. Laparoscopic sleeve gastrectomy as a revisional procedure for failed gastric banding: lessons from 300 consecutive cases. Surg Obes Relat Dis 2014;10:1116-22.

27. Silecchia G, Rizzello M, De Angelis F, Raparelli L, Greco F, Perrotta N, et al. Laparoscopic sleeve gastrectomy as a revisional procedure for failed laparoscopic gastric banding with a "2-step approach": a multicenter study. Surg Obes Relat Dis 2014;10:626-31.

28. Alqahtani AR, Elahmedi M, Alamri H, Mohammed R, Darwish F, Ahmed AM. Laparoscopic removal of poor outcome gastric banding with concomitant sleeve gastrectomy. Obes Surg 2013;23:782-7. 